\title{
Les grands enjeux de l'aménagement forestier durable au Québec
}

\author{
par Gilbert G. Paillé ${ }^{1}$
}

\section{Introduction}

Un colloque ${ }^{2}$ qui réunissait quelques centaines de représentants de tous les milieux du secteur forestier a été tenu en novembre 2000 pour analyser les enjeux de l'aménagement forestier intensif au Québec.

Les conférenciers ont traité de problèmes auxquels on doit faire face dans l'application de la sylviculture et ont présenté des projets pilotes. Pour notre part, on nous avait demandé d'idenปtifier les enjeux globaux de l'aménagement forestier intensif; Nnous avons préféré exposer les enjeux de l'aménagement forestier durable parce que nous assumions que, pour devenir Ẽou pour demeurer durable dans la plupart des régions, l'aménageఫment forestier devrait être intensifié de toute façon.

Cos enjeux ont été regroupés en sept catégories et présenftés en ordre décroissant d'importance. Aussi surprenant que cela puisse paraitre au premier abord, les enjeux forestiers proprement ثีdits se sont retrouvés au septième rang !

\section{Enjeux Politiques}

¿. Selon nous, quand il s'agit d'aménagement durable de 亩rêts publiques, il faut d'abord traiter des enjeux politiques.

\section{Sîlanque de reconnaissance officielle de l'extrême}

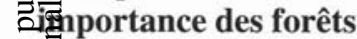

Z ¿đ̨̈lle de l'extrême importance économique, sociale et enviErínnementale de la forêt. Pour que la population finisse par reconE్fnâtre ce fait, on pourrait lancer des programmes massifs d'information et organiser un véritable lobby du secteur of forestier (qui n'existe pas encore à l'heure actuelle).

On pourrait aussi vouloir augmenter la visibilité de l'admiUnistration forestière publique en proposant la création de miJ̈nistères des Forêts tant au niveau provincial que fédéral (parce छ্ট̈que l'utilisation des forêts provinciales bénéficie aussi au gouvernement fédéral).

Toutefois, on peut penser que le chemin pour y arriver sera : II garante des succès pour l'avenir. En effet, sauf pour une périÆode de dix-huit mois au début des années 90' où il a été incorporé dans un ministère des Forêts, le Service forestier du Québec n'a pas souffert d'un surplus de notoriété depuis la Confédération et il a finalement été placé dans un ministère des Ressources naturelles qui s'occupe en même temps de l'Énergie, des Mines et des Terres ${ }^{3}$.

Nous sommes d'avis que tant qu'il n'y aura pas de volonté politique forte de reconnaître les forêts comme notre plus grande

\footnotetext{
${ }^{1}$ Ingénieur forestier, Ph. D., PDG FERIC, Montréal, QC.

${ }^{2} \mathrm{Ce}$ colloque était organisé par le Centre de Services aux Réseaux d'Entreprises du secteur forestier en collaboration avec le Ministère des Ressources Naturelles du Québec, la Conférence des coopératives forestières du Québec et l'Association des Manufacturiers de Bois de Sciage du Québec.
}

ressource naturelle et l'activité forestière comme notre plus grosse carte d'atout, on ne pourra pas garantir la durabilité de l'aménagement forestier, ni au Québec, ni au Canada.

\section{Manque de reconnaissance publique du besoin d'aug- menter l'intensité de l'aménagement forestier pour satisfaire la demande future}

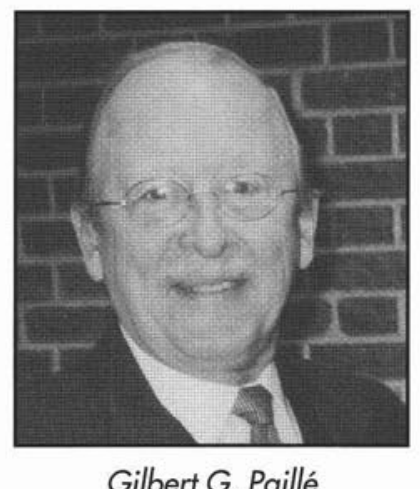

Le deuxième enjeu politique concerne le manque de reconnaissance par la population du besoin urgent d'augmenter l'intensité de l'aménagement forestier pour que la province puisse demeurer capable dans l'avenir de satisfaire la demande toujours croissante qui pèse sur les territoires forestiers et sur les ressources forestières qu'ils contiennent. Non seulement la population n'est pas convaincue qu'il faille augmenter l'intensité de l'aménagement, mais elle pense plutôt qu'il faudrait la diminuer !

Au cours des trente dernières années, la possibilité annuelle de coupe de toutes les forêts du Québec (publiques et privées) a déjà été augmentée de 45 pour-cent (passant de 38 à 55 millions de mètres cubes), suite à une augmentation considérable des traitements sylvicoles destinés principalement à accélérer

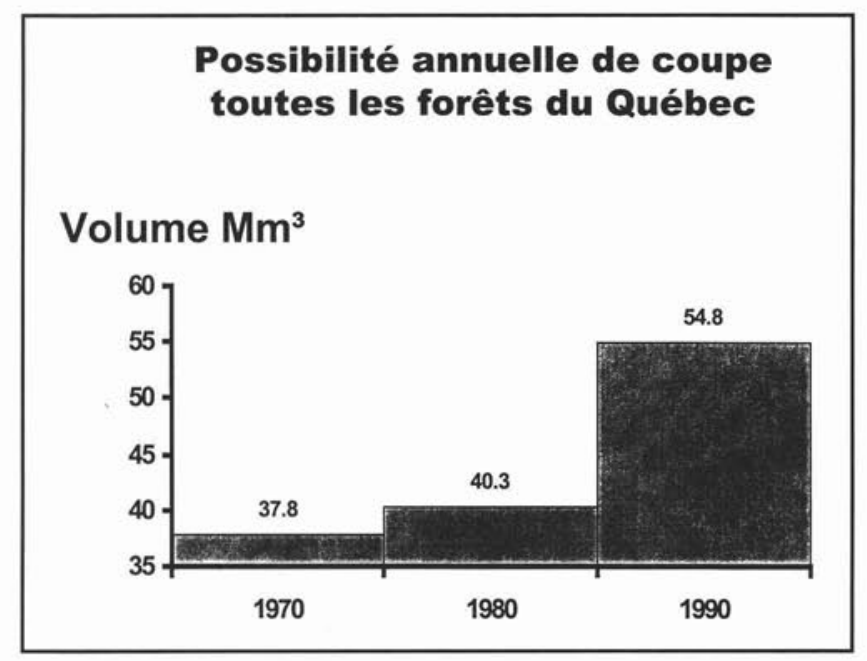

Fig. 1. 


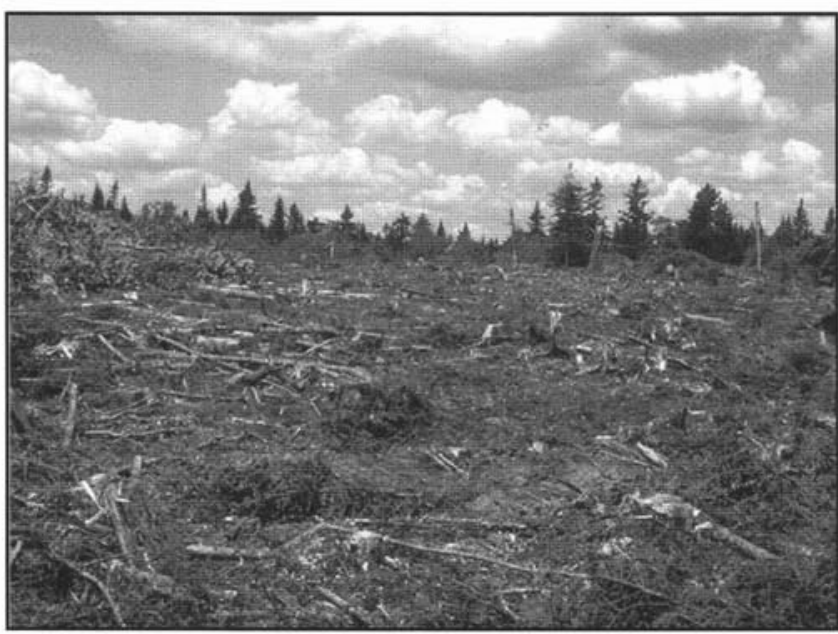

Fig. 2.

la régénération des parterres de coupe et à remettre en production ceux qui étaient tombés dans «l'arrérage » (Fig. 1). Pendant ce temps, le volume des récoltes annuelles de bois a toutefois été augmenté dans les mêmes proportions.

Or, l'accroissement de la pression de conservation qui est exercée sur les milieux forestiers d'une part et l'augmentation prévisible de la demande future de produits forestiers de consommation, créée principalement par l'augmentation de la population, font que le besoin d'intensifier l'aménagement forestier grandit rapidement.

Il est cependant difficile de prédire en longue période le rythme de l'augmentation de la demande pour chaque catégorie de produit ou l'endroit précis où la pression s'exercera. Or, ce sont essentiellement les deux éléments qui peuvent remettre la durabilité en cause dans plusieurs régions.

Pour avoir une chance d'améliorer la situation, on pourrait monter rapidement de vastes programmes d'information du public, mais il nous semble qu'on n'est pas vraiment prêt à identifier ce problème comme étant assez urgent pour justifier une injection massive de fonds publics pour ce faire.

\section{Enjeux Sociaux}

Après les enjeux politiques, les enjeux sociaux sont, selon nous, les plus importants.

\section{Objection de la population aux pratiques d'aménage- ment de la forêt}

De nos jours, la seule mention du mot «forêt» soulève immédiatement le spectre de la coupe à blanc et de son lot de préjugés associés (Fig. 2). De là à conclure au saccage des vieilles forêts et à l'expansion des monocultures, il n'y a qu'un pas.

Les gens croient à tort qu'il serait facile d'obliger l'industrie à effectuer des coupes partielles (pratiquement invisibles à l'oeil nu) dans toutes les forêts (même équiennes surannées), et à planter une multitude d'espèces (même si le reboisement n'est pas nécessaire ou que les espèces ne sont pas adaptées aux sites) pour maintenir la biodiversité. En somme, la population s'objecte aux pratiques traditionnelles d'aménagement forestier et, en plusieurs endroits, elle interdit même l'accès de la forêt aux ouvriers forestiers.
L'enjeu ici consiste à faire changer la perception négative qu'ont les gens des activités forestières de récolte et de régénération en milieu forestier afin que l'industrie forestière puisse obtenir l'autorisation sociale (social license) de pratiquer la foresterie sur une base durable partout où c'est possible et économique de le faire.

Ceci semble être une tâche considérable et un défi presque impossible à relever en plusieurs endroits du pays. Au Québec, il est probablement encore plus facile de le faire qu'ailleurs au Canada où les arbres sont plus gros, plus vieux ou plus visibles à cause de la topographie. Mais, on hésite à commencer !

\section{Conviction de la population que les entreprises forestières surexploitent la forêt}

C'est un mythe tenace que celui de la surexploitation des forêts. Le premier Premier ministre du Canada, J.A. Macdonald, en voyant descendre les billots qu'on transportait par flottage sur la rivière des Outaouais pensait en 1867 que cette activité ne pouvait pas durer. Le premier Premier ministre canadienfrançais, Wilfrid Laurier, s'inquiétait aussi en 1906 de la conservation des forêts de l'Est du Canada. En 1954, un des premiers scientifiques forestiers québécois formé aux États-Unis, Dr P.E. Lachance, démontrait qu'il y aurait eu alors surexploitation du bois à pâte dans onze groupes de bassins hydrographiques du Québec sur un total de vingt. En 1985, dans son Livre Blanc, le ministère de l'Énergie et des Ressources proposait un nouveau régime forestier en prenant tous les moyens pour éviter la surexploitation des forêts. La récolte de bois résineux (SEPM) dépassait alors, disait-on, la possibilité annuelle de coupe dans six régions administratives sur neuf en forêt publique et dans six territoires de syndicats de producteurs de bois sur dixhuit en forêt privée.

En 2000, la situation était rétablie sur papier, mais le ministre des Ressources naturelles, lors des audiences publiques sur le projet de modernisation du Régime forestier, parlait de la nécessité du rendement accru pour empêcher la surexploitation.

Le fait est que, de nos jours, le volume de bois sur pied dans les forêts du Québec est plus grand qu'il n'a jamais été (4 milliards de mètres cubes) et que plus de $50 \%$ des forêts publiques ont atteint ou dépassé la maturité sans que personne ne soit encore intervenu pour les récolter. En ce moment, il ne nous semble donc pas que ce soit la durabilité de l'ensemble de la forêt qui soit menacée (il y a des forêts naturelles partout où l'agriculture a été abandonnée ou n'est pas pratiquée) mais plutôt la disponibilité régionale de certaines essences présentant des caractéristiques déterminées.

La population aurait avantage à être mieux informée de la situation réelle, région par région, et les ajustements dans les prélèvements auraient avantage a être faits rapidement lorsque nécessaire (ceci est déjà commencé dans certaines régions du Québec et dans d'autres provinces du Canada).

\section{Conviction de la population que le travail forestier n'offre pas de bonnes perspectives d'avenir}

Une autre croyance populaire menace la durabilité de l'aménagement forestier par manque de main d'oeuvre qualifiée. On assume en effet assez généralement que le travail forestier requiert de la force physique mais peu ou pas de compétence spéciale (comme anciennement), qu'il est mal rémunéré et qu'il n'est pas une activité enviable. 
Il nous semble qu'on aurait grand avantage a faire mieux connaître dans la population la vraie nature du travail forestier moderne et les vrais défis qu' ont à relever les travailleurs de façon à assurer la relève et, par conséquent, la durabilité des activités en forêt.

La plupart des technologies modernes sont déjà entrées en forêt (hydraulique, informatique, télématique, robotique, navigation et positionnement par satellites, etc.) et elles doivent être maîtrisées par les opérateurs des équipements s'ils veulent en tirer le meilleur parti possible. On est bien loin de la hache, du «buck saw» et de la poche de farine ! Mais ce fait est encore mal connu.

\section{Enjeux Économiques}

Après les enjeux politiques et sociaux, il y a aussi les enjeux méconomiques qui menacent la durabilité de l'aménagement तुforestier.

Croyance populaire que l'utilisation industrielle de la छforêt profite surtout aux grosses compagnies et à leurs ‡actionnaires

Les gens qui croient que les plus gros et, dans plusieurs cas, fles seuls bénéficiaires de l'activité industrielle forestière sont -les grandes corporations à but lucratif et leurs actionnaires sont indans l'erreur. Ils ne tiennent pas compte du fait que l'industrie z̧forestière contribue plus à l'économie provinciale (balance comonerciale de 10 milliards de dollars en 1999) que plusieurs autres Sisecteurs économiques réunis. Ces dollars servent à supporter Fdécs dépenses comme celles de la santé, de l'éducation, des pro'Jggammes sociaux et des infrastructures à un niveau qui est même ôstapérieur à celui qu'on consacre aux forêts.

Z̃ En plus des devises étrangères, les entreprises du secteur forestige:

¿Les corporations forestières gagneraient beaucoup à faire conŻnîître aux populations locales et à leurs propres employés la "ूnthature exacte de leurs contributions aux économies régionales, provinciales et nationale dans le but de gagner plus de support opopulaire. Certaines formes de participation des populations Orégionales aux bénéfices des entreprises existantes conOtribueraient aussi grandement à rallier plus de support popu$\frac{3}{3}$

\section{Manque d'incitatifs économiques à l'augmentation du ت̛̣rendement des forêts} gouvernements provinciaux n'ont pas encore trouvé le Imoyen d'inciter les industriels forestiers à investir leur argent pour accroître le rendement futur des forêts qui ne leur appartiennent pas.

Les gouvernements provinciaux, en qualité de propriétaires des forêts publiques, ont commencé à réinvestir une partie des redevances payées par l'industrie sur les bois récoltés pour accélérer la régénération des peuplements et pour raccourcir les révolutions (temps nécessaire à une forêt pour se régénérer et pour atteindre la maturité). Ils investissent aussi un peu d'argent en forêt privée pour encourager les propriétaires fonciers à bien faire.

Mais les industriels qui détiennent des droits d'approvisionnement de leurs usines à long terme en forêts publiques ne sont pas encore certains de pouvoir récupérer à terme le fruit de leurs efforts. Dans l'incertitude, et comme de bons locataires,

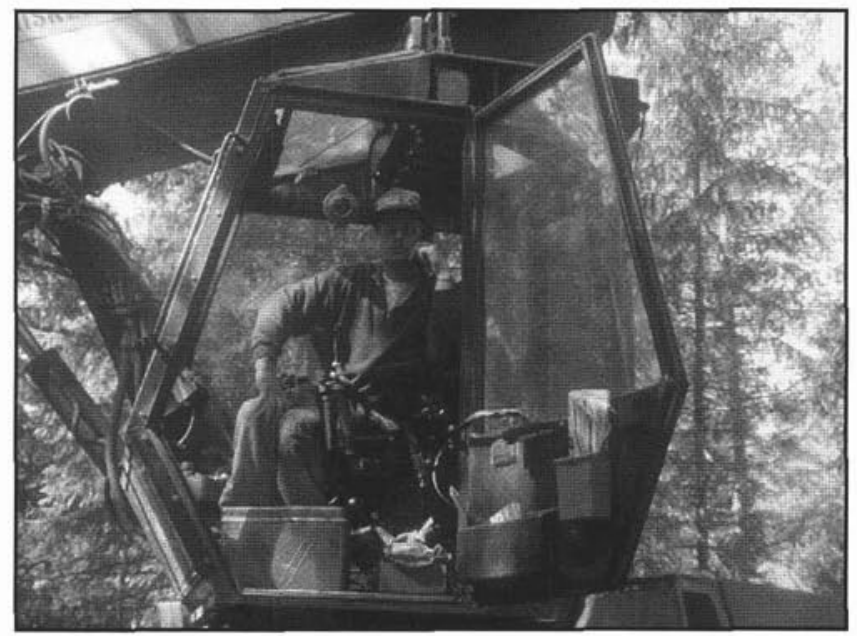

Fig. 3.

ils payent leur loyer mais ils s'abstiennent d'investir dans l'amélioration de l'immeuble qu'ils habitent !

Pour solutionner ce problème et garantir la durabilité du développement, le gouvernement pourrait, par exemple, penser à vendre le droit au rendement accru de telle forêt à l'industriel qui aurait investi pour l'obtenir.

\section{Obligation de maintenir le coût d'approvisionnement en fibres des usines au minimum}

Le troisième enjeu économique crucial pour les industries forestières, qui désirent sauvegarder leur capacité concurrentielle à l'échelle internationale et participer au développement économique de façon durable, consiste à maintenir leurs coûts d'approvisionnement en fibres à un minimum absolu, maintenant et dans le futur.

Pour ce faire, elles doivent rechercher les moyens d'économiser au maximum la fibre disponible dans chaque territoire en plus du temps et de l'énergie qui sont nécessaires pour effectuer les activités forestières. Seule l'utilisation constante des meilleures technologies de récolte, de transport et de protection de l'environnement peut leur permettre d'atteindre cet objectif (Fig. 3).

Le Chili, le Brésil et la Nouvelle-Zélande surveillent toutes les issues et sont prêts à livrer bataille dans le champ de l'approvisionnement à bon prix, principalement à cause de leurs structures de coûts et aussi des taux de croissance de la forêt qu'ils observent chez-eux.

\section{Enjeux Commerciaux}

En plus des enjeux économiques, nos entreprises forestières doivent aussi faire face à plusieurs enjeux commerciaux.

\section{Concurrence de tous les pays exportateurs de produits forestiers du monde}

Le Canada, étant le plus grand pays exportateur de produits forestiers du monde, doit faire face à la concurrence de tous les autres pays exportateurs qui veulent lui ravir des parts de marché. Ceci constitue une menace permanente au développement durable.

Nous croyons que le maintien de cette position privilégiée passe par la réduction des barrières tarifaires sur les produits 
forestiers, non seulement à l'échelle des Amériques (l'ALENA constitue une protection durable), mais aussi à l'échelle mondiale.

Il est peut-être encore plus important de travailler d'arrachepied pour combattre les barrières non tarifaires à l'importation de produits forestiers imposées par les pays acheteurs pour protéger leurs propres marchés.

\section{Manque de débouchés pour les bois feuillus de trituration}

Le manque de débouchés commerciaux pour les bois feuillus de trituration empêche la réalisation de l'aménagement forestier dans les forêts feuillues et mélangées du sud du Québec, donc le développement durable.

Comme ces bois peuvent servir de bois de chauffage (sous forme solide ou sous forme de charbon de bois) et qu'il existe une pénurie mondiale de cette denrée, on pourrait peut-être envisager d'en offrir à titre d'aide humanitaire aux pays en développement qui en ont le plus besoin.

\section{Manque d'intérêt des industriels pour la propriété fon- cière et manque d'intérêt des propriétaires fonciers pour l'industrie}

Il y a 1375 usines de transformation primaire du bois au Québec. Elles sont pour la plupart situées dans le domaine des forêts privées qui sont détenues par quelque 120000 propriétaires individuels.

Or, on constate qu'un nombre infime de propriétaires d'usines possèdent leurs «limites à bois». Par ailleurs, on constate aussi qu'un nombre infime de propriétaires de boisés détiennent des intérêts dans l'industrie forestière, même celle qui a un caractère régional. Pour nous, ce paradoxe constitue un enjeu commercial de taille pour lequel il faut trouver des solutions.

Une de celles-ci pourrait consister pour les propriétaires terriens à offrir des contrats d'usufruit forestier aux industriels qui ont besoin de bois ; en échange, les industriels qui s'approvisionnent des boisés privés pourraient offrir des participations dans leurs entreprises aux propriétaires de ces boisés.

La première conséquence de cette symbiose serait certainement d'augmenter le degré de sécurité d'approvisionnement des industries, de faire baisser les coûts de la matière première et d'augmenter la stabilité du système production-consommation, c'est-à-dire de favoriser le développement durable.

\section{Enjeux Environnementaux}

Les enjeux environnementaux constituent une catégorie d'enjeux dont l'importance n'a cessé d'augmenter au cours des vingt-cinq dernières années.

\section{Conviction de la population que toutes les grandes usines sont polluantes}

Le premier enjeu environnemental concerne la croyance bien ancrée dans la population que toutes les grandes usines sont polluantes. Comme la plupart des scieries et des papeteries sont de grandes usines, situées en zones habitées, à proximité des principaux cours d'eau, on croit généralement que leurs cheminées crachent des fumées polluantes dans l'air et que leurs rejets liquides polluent l'eau des rivières à un niveau inacceptable. Donc, elles sont indésirables. Or le développement économique ne peut se faire de façon durable sans usines.

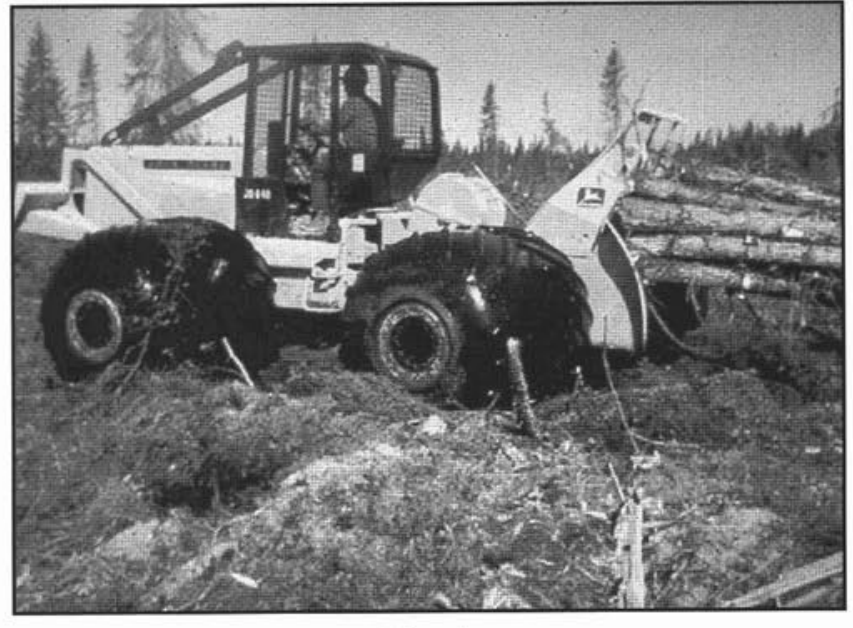

Fig. 4.

Les industries forestières auraient avantage à mieux informer la population des progrès accomplis dans le nettoyage de leurs rejets et à comparer le secteur forestier avec les autres secteurs industriels.

\section{Conviction de la population que la machinerie forestière détruit la forêt}

Le deuxième enjeu environnemental concerne aussi une croyance bien ancrée dans la population selon laquelle la (grosse) machinerie forestière perturbe sérieusement les sols forestiers, la nappe phréatique et la régénération, quand elle ne pollue pas carrément l'eau des ruisseaux et des rivières. Or l'aménagement forestier moderne ne peut pas se pratiquer sans machineries (Fig. 4).

Les règlements du gouvernement concernant la protection de l'eau, du sol et de la régénération sont parmi les plus sévères en Amérique du Nord. Dans plusieurs cas, ils sont aussi sévères sinon plus que dans le domaine agricole. De plus, la plupart des machines utilisées en forêt proviennent de pays où les préoccupations environnementales sont aussi grandes sinon plus grandes qu'au Canada, comme aux États-Unis, au Japon, en Finlande et en Suède.

La population gagnerait beaucoup à être mieux informée des règlements du gouvernement concernant la protection de l'environnement forestier et des caractéristiques des équipements forestiers, de leurs modes d'utilisation et de leurs effets réels sur l'environnement.

\section{Conviction de la population qu'il est écologiquement préférable d'utiliser des matériaux de remplacement plutôt que du bois}

Un troisième enjeu environnemental qui devient de plus en plus important avec le temps est la conviction d'une proportion croissante de la population qu'il est plus écologique d'utiliser le plastique, l'aluminium, l'acier ou le béton que le bois ! (Fig. 5).

Les grandes campagnes de conservation des forêts (tropicales) menées par les environnementalistes ont pour conséquence, entre autres, de provoquer chez ceux qui utilisent du bois pour satisfaire leurs besoins personnels un sentiment de culpabilité. Et pourtant, de tous les matériaux utilisés par l'homme, le bois 


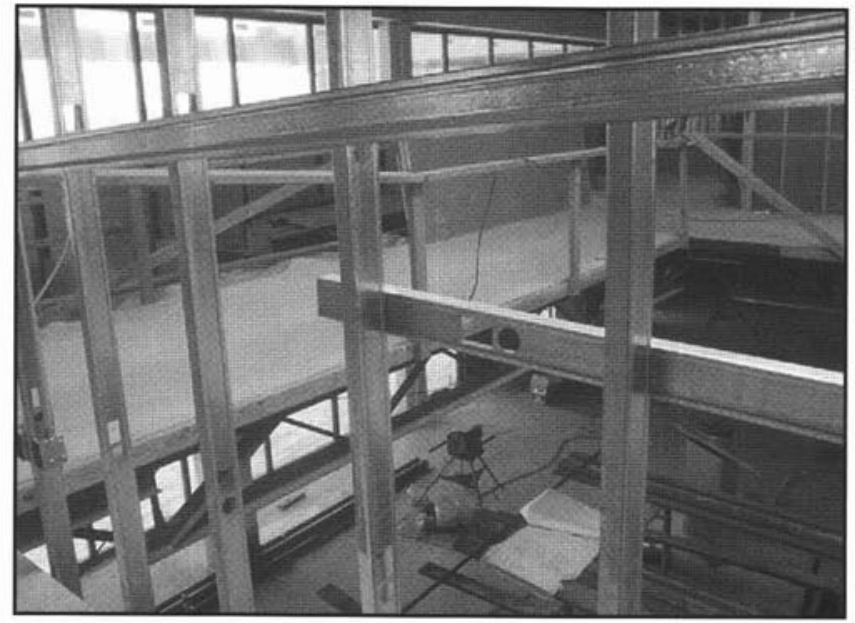

Fig. 5.

est celui qui est le plus écologique ; il est produit à l'énergie solaire ; il est biodégradable et recyclable ; il provient de forêts qui croissent et se régénèrent naturellement.

Par comparaison, les principaux matériaux de remplacement sont fabriqués à l'aide de combustibles fossiles, de matières premières non renouvelables, ne sont pas biodégradables et ne sont que partiellement recyclables. Mais, on en fait une promotion extrêmement agressive, supportée par des ressources de cinq à vingt-cinq fois plus considérables que celles qu'on utilise pour promouvoir l'utilisation du bois. Conséquence : le bois perd du terrain ! Or, en présence d'une demande décroissante pour le bois, il ne peut y avoir d'aménagement forestier durable.

Ici encore, le moyen de relever cet enjeu passe, selon nous, par des programmes d'information de la population mieux ciblés et mieux supportés. Des initiatives du type «Wood is Good» doivent être fortement encouragées.

\section{Enjeux Territoriaux}

Avant de parler des enjeux forestiers, il faut parler des territoires sur lesquels poussent les forêts. Nous pouvons identifier ici trois enjeux.

\section{Droits ancestraux des autochtones mal définis et mal connus}

Le premier enjeu territorial concerne les droits ancestraux des Autochtones sur une grande partie des territoires forestiers du Québec et du Canada. La reconnaissance de ces droits pourrait avoir pour conséquence de soustraire une partie importante des forêts publiques à l'aménagement forestier industriel, avec un impact direct sur la durabilité des développements sur les territoires restants.

Il faudrait favoriser une définition claire des droits ancestraux des Autochtones sur les territoires forestiers dans les meilleurs délais et tenter par des ententes de gestion de préserver la vocation de production industrielle de la matière ligneuse de ces territoires, en plus des intérêts de ceux qui les habitent.

\section{Empiétement sur les territoires affectés prioritairement à la production de matière ligneuse \\ La superficie des terres forestières au Québec est immense : 84 millions d'hectares ou 840000 kilomètres carrés. Mais la}

superficie des terres forestières productives et accessibles au sud du 52e parallèle (reconnue comme la limite nordique des forêts commerciales) est déjà beaucoup moins grande : 52 millions d'hectares. Si de ce total on soustrait les terres privées et les terres fédérales, les terres forestières publiques sont réduites à 45 millions d'hectares. Lorsqu'on tient compte de leur affectation, celles qui sont réservées pour la production forestière prioritaire ne couvrent plus que 33 millions d'hectares.

Or depuis le dépôt en 1988 du rapport de la Commission mondiale sur l'environnement et le développement (Brundtland), on vise partout à protéger $12 \%$ du territoire pour la postérité. Au Québec, les aires protégées s'étendent maintenant sur 4,7 millions d'hectares (ou 2,8\% du territoire) (Fig. 6); lorsqu'on aura atteint $12 \%$, la superficie protégée sera de 20 millions, c'est-à-dire une superficie équivalente aux deux-tiers de celle qui est maintenant affectée à la «production forestière prioritaire»! L'impact véritable des aires protégées sur le développement forestier durable ne sera connu que lorsqu'elles seront localisées. Mais, à terme, il sera considérable.

Par ailleurs, les terres agricoles sont zonées vertes au Québec, mais les forêts ne le sont pas ! Il est donc plus facile d'y empiéter pour établir les aires protégées ou pour les fins de l'industrialisation, de l'urbanisation ou de l'agriculture. Nous croyons que le manque de protection du territoire dédié en permanence à la production forestière est un désincitatif puissant qui empêche les investissements dans l'aménagement par les compagnies forestières et même par le gouvernement, mettant la durabilité du développement en danger dans plusieurs régions.

Pour que l'aménagement forestier puisse être certifié et être durable, il nous semble très urgent que des Unités Forestières de Référence (UFR) soient bien délimitées et qu'on prenne les moyens pour que ces limites deviennent immuables (comme les clôtures qui délimitent les propriétés des agriculteurs). À cette fin, un consensus devra être établi entre les mandataires du secteur forestier pour déterminer la nature des limites géographiques qui seront retenues.

En ce qui nous concerne, nous suggérons que les 102 bassins hydrographiques du Québec deviennent les UFR. Ce sont des territoires dont les limites sont immuables parce qu'elles correspondent avec les hauteurs de terre et ce sont des territoires envers lesquels les Québécois qui les habitent entretiennent un fort sentiment d'appartenance, donc un grand désir d'y assurer le développement durable.

D'autres proposeront sans doute qu'on retienne les limites des Comtés, des MRC, des Unités de Gestion du MRNQ ou des Aires Communes. Le gouvernement aura le dernier mot.

\section{Superposition des droits d'usage}

L'aménagement intégré des ressources naturelles renouvelables constitue un objectif général à atteindre dans toutes les forêts publiques du Québec. Il consiste à aménager toutes les ressources du milieu en permettant tous les usages. Pour le gouvernement, ceci se fait normalement en attribuant à divers bénéficiaires des tenures et des droits d'usage différents qui, dans bien des cas, se superposent au même endroit. Par exemple, les quelque trois cents détenteurs de contrats d'approvisionnement et d'aménagement forestier (CAAF) détiennent des droits sur les bois nécessaires pour approvisionner leurs usines ; ces bois sont situés dans des Aires Communes qui s'étendent 


\section{Évolution de la superficie et du nombre d'aires protégées au Québec au cours des 30 dernières années}

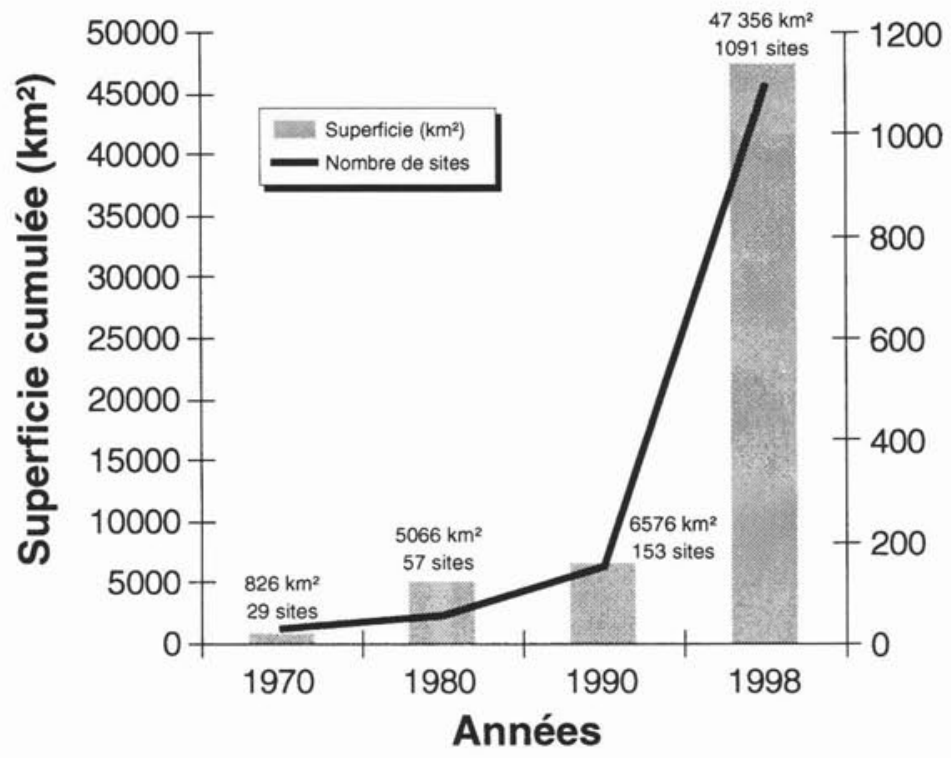

Fig. 6.

La progression significative observée entre 1990 et 1998 s'explique par l'ajout de 693 habitats fauniques $\left(38277 \mathrm{~km}^{2}\right.$ ) ainsi que 162 rivières à saumons (bande riveraine). Toutes les aires fauniques ( $38277 \mathrm{~km}^{2}$ ) ainsi que 162 rivières à saumons
protégées (catégories I à VI de I'UICN) sont considérées.

Source: Centre de données sur le patrimoine naturel du Québec, 1999.

sur quelque 30 millions d'hectares de forêts productives commerciales. À certains endroits à l'intérieur des Aires Communes on retrouve jusqu'à seize détenteurs de droits sur différentes essences forestières ou sur différents produits forestiers.

Pour les fins de la gestion de la faune, le territoire est aussi subdivisé en Zones d'Exploitation Contrôlées (ZEC)qui recouvrent quelque 4,8 millions d'hectares et en Pourvoiries de Chasse et de Pêche qui s'étendent sur quelque 2,4 millions d'hectares. Dans la majorité des cas, ces droits fauniques s'exercent dans les Aires Communes.

De plus, le gouvernement attribue aussi des baux de villégiature autour des lacs et rivières (zones forestières et récréatives) à ceux qui veulent se récréer en forêt.

Cette méthode de superposition des droits rend possible l'aménagement intégré des ressources forestières mais, en même temps, menace par endroits la durabilité des développements par manque d'ententes entre les détenteurs de tels droits.

Des processus de médiation efficaces doivent être adoptés, mis en place et acceptés par tous les intervenants pour assurer l'harmonie des activités économiques, la protection de l'environnement et le développement harmonieux et durable.

\section{Enjeux Forestiers}

Finalement, on en arrive aux enjeux forestiers. Nous sommes d'avis que l'aménagement forestier durable n'est possible qu'à la condition de bien définir : a) la stratégie d'aménagement des territoires forestiers à l'échelle provinciale, b) les objectifs de rendement forestier escomptés pour chaque Unité Forestière de Référence (UFR), c) la terminologie utilisée et la méthode de mesure des résultats attendus.

\section{Définition de la stratégie d'aménagement des territoires forestiers}

Avant la Confédération, la stratégie d'aménagement forestier adoptée au Québec était très simple. Elle consistait pour les entrepreneurs à récolter quelques espèces convoitées le long du Saint-Laurent et de ses principaux affluents.

Depuis ce temps, et jusqu'à l'implantation du Nouveau Régime Forestier en 1990, l'aménagement forestier était essentiellement réalisé sur des concessions forestières. Il consistait à récolter plusieurs espèces convoitées dans les peuplements mûrs seulement et en quantité égale ou inférieure à la possibilité annuelle de coupe. Celle-ci était calculée en tenant compte de la régénération naturelle de la forêt et du principe du rendement soutenu.

Depuis 1990, la demande de produits forestiers excède la capacité de production de la forêt naturelle. Pour tenter de la satisfaire sans mettre en péril la pérennité de la ressource, on oblige les industriels à pratiquer le reboisement pour assurer la régénération des forêts qui mettent trop de temps à se régénérer naturellement, l'éclaircie pré-commerciale pour raccourcir la période de révolution des jeunes peuplements résineux denses et le jardinage des forêts mélangées et feuillues.

Comme nous l'avons vu plus haut, la demande de ressources fauniques et récréatives augmente aussi en suivant la courbe d'augmentation de la population et, simultanément, la pression de conservation augmente de façon exponentielle.

Il est donc devenu impératif de choisir une stratégie d'aménagement forestier pour le Québec. Nous suggérons l'adoption d'une stratégie souple qui tienne compte de la nature des forêts et de leur potentiel. Ainsi, les forêts nordiques de 
conifères seraient aménagées extensivement pour la production de fibre en grande quantité tout en protégeant la biodiversité ; les forêts mélangées et feuillues du sud du Québec seraient aménagées intensivement surtout pour produire des bois de qualité ; finalement, les terres les plus fertiles situées autour des usines en majorité dans le domaine privé seraient dédiées à la ligniculture, c'est-à-dire à l'aménagement super intensif.

Toutefois, d'autres auteurs proposent des stratégies différentes. Le sous comité sénatorial sur la forêt boréale propose de consacrer $20 \%$ du territoire aux aires protégées, $20 \%$ à l'aménagement forestier intensif et $60 \%$ à l'aménagement écosystémique. Le professeur Messier pour sa part propose de consacrer $74 \%$ du territoire à l'aménagement écosystémique, $12 \%$ aux aires protégées, $10 \%$ à l'aménagement intensif et 4 $\%$ à la ligniculture.

Le gouvernement voudra sans doute faire participer tous les mandataires du secteur forestier au choix d'une stratégie d'aménagement durable pour le Québec car ils devront tous être impliqués dans sa mise en oeuvre.

\section{Définition des objectifs de rendement forestier escompté pour chaque unité forestière de référence}

Afin de faire respecter les critères du développement durable dans chaque UFR, il faudra par ailleurs que les intervenants conviennent d'y appliquer la portion de la stratégie provinciale d'aménagement choisie, et qu'ils s'entendent sur le niveau d'intensité de l'aménagement forestier souhaité. Une fois cette étape franchie, on pourra définir les activités d'aménagement les plus a appropriées pour atteindre l'objectif et s'entendre sur la distribution

\section{Définition de la terminologie utilisée et mesure des}

\section{Örésultats attendus}

En dernier lieu, il nous semble qu'il existe un grand besoin de définition et de vulgarisation de la terminologie utilisée. Même les spécialistes ont de la difficulté à s'entendre sur la signification de plusieurs termes comme l'aménagement intégré des ressources, l'aménagement écosystémique, l'aménagement intensif, le rendement soutenu, le rendement accru et la possibilité annelle de coupe, pour n'en nommer que quelques-uns. Une fois les définitions bien comprises par les intervenants et les résultats escomptés bien identifiés, il faudra encore convenir des méthodes de mesure des résultats attendus à court, à moyen et à long terme.

Finalement, restera à vulgariser le tout suffisamment pour que la population comprenne la marche à suivre vers le développement durable des forêts de chaque région forestière du Québec.

\section{Conclusion}

Voilà, selon nous, quels sont les principaux enjeux de l'aménagement forestier durable et quelles sont les activités les plus importantes à prévoir pour assurer qu'il se réalise.

Nous constatons que la responsabilité de la mise en oeuvre de la plupart de ces activités ne relève pas uniquement du ministère provincial des Ressources naturelles ou des ingénieurs forestiers ; elle implique tout le secteur forestier et toutes les communautés qui profitent des activités forestières. Les institutions financières, les organismes de recherche, les ONG, les environnementalistes et les populations régionales concernées doivent faire front commun avec les propriétaires de boisés, l'industrie forestière et le gouvernement provincial pour assurer la mise en oeuvre ordonnée de ces activités.

Lorsque, par des programmes massifs et soutenus d'information et de vulgarisation, nous aurons réussi à faire changer la perception négative des activités forestières industrielles dans la population en général, nous pourrons progresser vers l'aménagement forestier durable au Québec.

Nous n'avons pas la prétention d'avoir traité ici ce vaste sujet en profondeur. Notre intention était plutôt de mettre le défi de l'aménagement forestier en perspective. Les réactions spontanées des auditeurs nous ont démontré que nous avions assez bien réussi.

Reste à passer à l'action. 